\title{
OS IMPACTOS DA HIPNOSE DURANTE A GRAVIDEZ \\ E TRABALHO DE PARTO \\ CATEGORIA: CLÍNICO
}

\section{INSTITUIÇÃO: CENTRO UNIVERSITÁRIO SÃO CAMILO}

\section{AUTORES:}

Molina, CA ${ }^{1}$ - Rua Acuruá, 97 - Vila Romana. CEP 05053-000, (11)

96630-2806,.cmol.assis@gmail.com

Germino, C1.

\section{ORIENTADORA:}

Oliveira, DLS²

1 Discentes da Faculdade de Medicina do Centro Universitário São Camilo

2 Docente da Faculdade de Medicina do Centro Universitário São Camilo 
OS IMPACTOS DA HIPNOSE DURANTE A GRAVIDEZ

E TRABALHO DE PARTO

CATEGORIA: CLÍNICO

DESCRITORES: "HYPNOSIS"; "PREGNANCY" 


\section{RESUMO}

INTRODUÇÃO:A hipnose é um tipo de intervenção psicológica que tem sido aplicada em muitos ambientes diferentes, incluindo a obstetrícia. Acredita-se que essa prática leve a um estado mental natural podendo ser descrito como "um estado de consciência alterada" caracterizado por maior receptividade a sugestões e modificações de percepção e memória. Nesse contexto, destaca-se um maior potencial para controle sistemático de uma variedade de funções fisiológicas. Diante disso, estudos demonstram benefícios do uso da hipnose para alívio dos sintomas gestacionais e controle da dor no trabalho de parto.METODOLOGIA: O banco de dados PubMed foi consultado com os descritores "hypnosis" e "pregnancy". Um total de 477 artigos foram encontrados e 10 foram selecionados, sendo excluídos os artigos anteriores a 2013 e os artigos de revisão.OBJETIVOS: Avaliar a literatura acerca da prática de hipnose em mulheres grávidas, ao longo da gestação e durante as fases do trabalho de parto. RESULTADOS: Dentre os artigos selecionados, os grupos "casos" demonstraram significativa redução de ansiedade (OR 1,44) e sintomas físicos (OR 1,41) quando comparados aos grupos controle, principalmente durante o último trimestre de gestação. Observou-se também redução dos níveis de estresse ao longo da gestação. Em relação ao trabalho de parto, nenhum estudo demonstrou impacto do uso da hipnose em sua duração. No entanto, a solicitação por anestesia epidural foi significativamente menor naquelas mulheres que haviam sido submetidas à prática (19\% em comparação a $28 \%$ de mulheres não submetidas). Uma avaliação epidemiológica, concluiu que mulheres adeptas à hipnose durante a gestação são mais propensas a utilizarem outras formas de medicina complementar, além de sentirem-se menos dependentes da presença e das ações do obstetra durante o trabalho de parto.CONCLUSÃO: O estado mental natural proporcionado pela hipnose tem inúmeras aplicações, inclusive no contexto da medicina. Em relação à gestação e ao momento do trabalho de parto, destaca-se que a hipnose pode influenciar nos sintomas físicos, psicológicos e comportamentais vivenciados pelas mulheres. No momento do parto, a hipnose demonstrou atuação no alívio da dor e controle de estresse, evidenciando que esse tipo de intervenção pode complementar a medicina tradicional, auxiliando mulheres ao longo da gestação. DESCRITORES: "HYPNOSIS"; "PREGNANCY" 


\section{INTRODUÇÃO:}

A hipnose é um estado alterado da consciência, induzido pelo procedimento "hipnotismo"1 e que atualmente é utilizada como intervenção psicológica, sendo aplicada em diversos ambientes, incluindo a obstetrícia.

Esse estado alterado da consciência deriva da capacidade individual de a pessoa conseguir ou não entrar num estado hipnótico. Logo, segundo Martin citando Barber, os indivíduos conseguem ser divididos em três grupos:

- O grupo de pessoas mais HIPNOTIZÁVEIS "pessoas que tendem a fantasiar com frequência no seu dia-a-dia"1

- O grupo de pessoas que tendem a ter AMNÉSIA "havendo inclusivamente [sic] fragmentos da sua vida fora da sua memória"1

- O grupo seria de pessoas com "elevadas expectativas, motivação e atitudes positivas face à hipnose"1, o que podemos inferir ser o grupo com maiores expectativas frente à hipnose.

Martin cita as diversas definições aplicadas durante os anos a respeito da prática do hipnotismo:

"É um sono desperto guiado: o hipnotizador (hetero-hipnose) ou o próprio sujeito (auto-hipnose) atua como guia para uma experiência considerada fantasiosa (Barrett, 1979).

É um estado alterado de consciência natural: a pessoa que está num estado de consciência diferente do "normal", chamado hipnose, através dum processo natural que não implica a ingestão de substâncias ou outros procedimentos psicológicos (Tart, 1972).

É um estado de relaxamento hiper-sugestionável: a pessoa entra num estado de relaxamento muito elevado, tanto mental como corporal, com a consequente descida de vigilância, o que propicia uma maior resposta à sugestão (Edmonston, 1991).

É um estado dissociado: envolve desconexão entre os processos conscientes e inconscientes, o que permite tornar a parte inconsciente mais acessível e recetiva às sugestões (Hilgard,1977)" 1

Através dessas definições de hipnose, podemos inferir que é um "estado de consciência alterada" e que proporciona a sensação de relaxamento, sem a utilização 
de qualquer substância, e esse estado pode ser atingido de maneira individual (autohipnose) ou guiado por um hipnotizador.

Martin também cita a American Psychological Association, definindo a atuação da hipnose no indivíduo:

\begin{abstract}
"A indução hipnótica é uma sugestão, extensa e ampla para usar a própria imaginação, que se dá no começo, e que pode incluir maiores elaborações da introdução. Usa-se um procedimento hipnótico para fomentar e avaliar respostas às sugestões. Ao usar a hipnose, uma pessoa (o sujeito) é guiada por outra (o hipnotizador) para que responda às sugestões de mudanças na experiência subjetiva, alterações na perceção, sensação, emoção, pensamento ou conduta. As pessoas podem aprender também autohipnose, que consiste em administrar procedimentos hipnóticos a si mesmo. Se o sujeito responde às sugestões hipnóticas, geralmente infere-se que foi induzido em hipnose."1
\end{abstract}

Nesse contexto, destaca-se o potencial de aplicabilidade da indução hipnótica em uma variedade de funções fisiológicas. Essas funções são demonstradas por diversos estudos, e dentre eles, está descrito o uso da hipnose para alívio dos sintomas gestacionais e controle da dor no trabalho de parto.

\title{
OBJETIVOS:
}

O objetivo do presente trabalho é avaliar a literatura acerca da prática de hipnose em mulheres grávidas, ao longo da gestação e durante as fases do trabalho de parto. Possuindo como objetivos específicos:

- Avaliar evidências sobre o uso da prática de hipnose no trabalho de parto para alívio de dor, estresse e ansiedade.

- Avaliar evidências sobre o uso da prática de hipnose durante a gestação para alívio de sintomas físicos, estresse e ansiedade.

- Identificar de quais formas a hipnose tem sido aplicada em gestantes.

\section{MÉTODOS:}

Foi realizada uma revisão de literatura, não sistemática, através de estudos primários, consultando o banco de dados PubMed. Os descritores utilizados foram: "hypnosis" e "pregnancy". A busca resultou num total de 477 artigos. 
Como critérios de inclusão temos: ano de publicação entre 2013 e 2018. E critérios de exclusão: estudos que consistiam em revisões sistemáticas, com ou sem metanálise, revisões não sistemáticas, guidelines, análises de decisão, análises econômicas, além de adequação ao objetivo do trabalho, sendo os artigos que não possuíam hipnose como intervenção em pacientes gestantes. A busca resultou num total de 10 artigos, como podemos perceber no fluxograma presente na FIGURA 1.

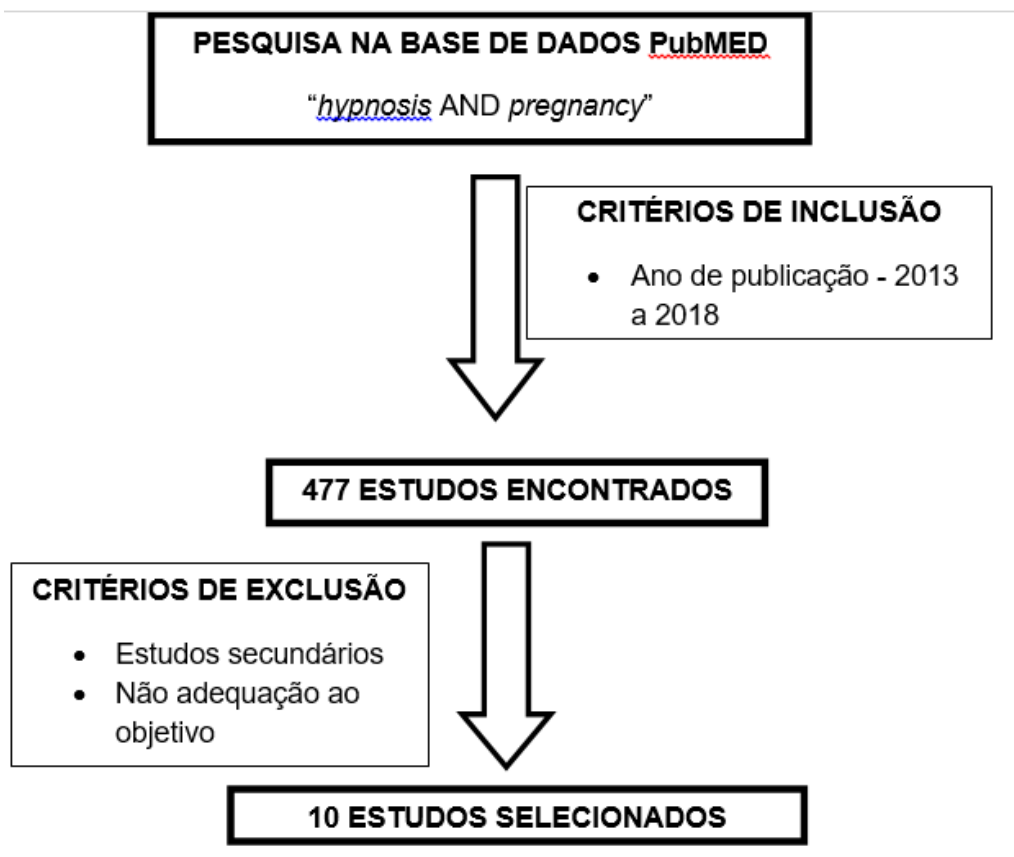

FIGURA 1: FLUXOGRAMA DE METODOLOGIA.

\section{RESULTADOS E DISCUSSÃO:}

A experiência de dor no parto é uma vivência subjetiva e variável, assim como a gestação que, ao longo dos trimestres, abrange inúmeros e diferenciados sintomas, sensações e sentimentos. Nesse âmbito, estudos são categóricos ao afirmar que a maioria das mulheres preferiria passar pelo momento do trabalho de parto sem nenhum tipo de intervenção médica, incluindo analgesia farmacológica. (2) Contudo, o que é observado na prática é que a anestesia epidural é a analgesia mais frequentemente utilizada (atualmente em cerca de $30 \%$ dos nascimentos no Reino Unido e em mais de $60 \%$ nos Estados Unidos). ${ }^{(3)}$

Ainda que seja considerada a forma mais efetiva para alívio da dor no trabalho de parto, os estudos destacam que o uso da epidural não necessariamente se associa 
a altos níveis de satisfação. ${ }^{(3)}$ Isto é, observou-se que aquelas mulheres que usam analgesia epidural, de modo geral, apresentam-se menos satisfeitas com sua experiência de trabalho de parto e nascimento em comparação com aquelas que não utilizam a anestesia. (2) Ademais, tanto a epidural quanto as outras opções farmacológicas apresentam uma vasta gama de efeitos colaterais: nulíparas que receberam epidural apresentaram risco aumentado para necessidade de intervenções, com o risco de morbidade associada e custos adicionais; já em relação a analgesia narcótica, segunda mais comumente utilizada, observou-se ineficácia no alívio da dor e associação com efeitos neonatais adversos. ${ }^{(3)}$

Nesse cenário, a expectativa pelo nascimento, por possíveis intercorrências, pela dor das contrações e até mesmo pela dor da anestesia desenha um ambiente de extremo estresse e ansiedade.(4) Diante disso, muitas mulheres iniciam com antecedência a busca por estratégias as auxiliem nesse momento de maior tensão, contribuindo para redução dos sintomas psicológicos.

Em resposta a essa crescente demanda, os prestadores de serviços públicos e privados estão oferecendo técnicas alternativas para o parto e para o alívio da dor, incluindo programas focados na prática de hipnose. ${ }^{(3)}$

Waisblat et al, testou a hipnose em mulheres que solicitaram a anestesia epidural com intuito de reduzir a ansiedade e medo durante o processo.5 Com uma abordagem cega, contou com uma equipe de anestesistas treinados em hipnose clínica que, durante a aplicação da anestesia, utilizou uma estratégia de comunicação positiva e sugestiva. Foi observada uma redução de até $20 \%$ no estresse ao longo de todas as fases do trabalho de parto e também na intensidade da dor das contrações. ${ }^{(5)}$

Os outros estudos avaliados optaram por uma intervenção anterior ao momento do parto, com seções de hipnose ao longo da gestação. Beevi et al, adotou uma prática com quatro sessões de hipnose focadas na redução de sintomas psicológicos e aumento do relaxamento muscular para uma experiência positiva de trabalho de parto e pós-parto.(6) $\mathrm{Na}$ terceira e quarta sessões incorporaram sugestões sobre uma experiência positiva de trabalho de parto e pós-parto visando menor desconforto durante o parto e vínculo positivo das mulheres com seus bebês recém-nascidos, (incluia orientar os participantes para o futuro, pedindo-Ihes para imaginar estar em trabalho no hospital e o tempo após a conclusão do trabalho de parto).6 Nesse 
contexto, no momento do parto, observou-se que a solicitação por anestesia epidural foi significativamente menor naquelas mulheres que haviam sido submetidas à prática de hipnose $\left(4,3 \%\right.$ versus $0 \%$ de mulheres submetidas). ${ }^{(6)}$

Essa redução na solicitação por anestesia epidural foi o resultado mais consistentemente observado nos estudos avaliados: $28 \%$ versus $31 \%$ de mulheres submetidas em Downe et al, $72 \%$ versus $81 \%$ em Finlayson et al e $30 \%$ versus 12,5\% em Beevi et al. ${ }^{(2,3,4)}$

Ademais, em relação aos sintomas psicológicos ao longo dos trimestres gestacionais, Downe et al demonstrou significativa redução de ansiedade $(O R$ 1,44) e sintomas físicos $(\mathrm{OR} 1,41)$ nas pacientes em uso da hipnose quando comparadas ao grupo controle, principalmente durante o último trimestre.(3) Já Streibert et al, evidenciou um impacto do uso de hipnose na indução de sentimentos mais positivos em relação à perspectiva do momento do parto e também uma maior facilidade no vínculo materno-fetal nos primeiros meses.

Nesse âmbito, destaca-se a dificuldade em se apurar os resultados de estudos lidando com uma prática tão subjetiva. Diante disso, evidencia-se a necessidade de novas pesquisas que possam fomentar o interesse dos serviços e profissionais de saúde, visando maior oferta de práticas complementares. ${ }^{(7)}$

\begin{tabular}{|c|c|c|c|}
\hline AUTOR & ANO & TIPO DE ESTUDO & RESULTADOS \\
\hline Werner A, et al. & 2013 & Caso-controle & $\begin{array}{l}\text { - Redução do tempo de trabalho de parto em até } 60 \text { minutos no grupo que utilizou hipnose. } \\
\text { - No grupo que utilizou hipnose foram registradas menos cesáreas eletivas. } \\
\text { - No periodo de puerpério as mulheres que fizeram uso de hipnose se queixaram de menos dificuldades no cuidado com a criança. }\end{array}$ \\
\hline Cyna AM, et al. & 2013 & Caso-controle & $\begin{array}{l}\text { - Nenhuma diferença foi observada em relação à intensidade de dor referida durante o trabalho de parto nos dois grupos. } \\
\text { - Nenhuma diferença foi observada em relação à necessidade e escolha de analgesia durante o trabalho de parto nos dois grupos. }\end{array}$ \\
\hline $\begin{array}{l}\text { Streibert LA, et } \\
\text { al. }\end{array}$ & 2015 & Coorte & $\begin{array}{l}\text { - Foi demonstrado um impacto do uso de hipnose na indução de sentimentos mais positivos em relação à perspectiva do momento do } \\
\text { parto. }\end{array}$ \\
\hline Downe S, et al. & 2015 & Caso-controle & $\begin{array}{l}\text { - Significativa redução de ansiedade }(\mathrm{OR} 1,44) \text { e sintomas físicos (OR } 1,41) \text { nas pacientes em uso da hipnose quando comparadas ao } \\
\text { grupo controle, principalmente durante o último trimestre de gestação. } \\
\text { A solicitação por anestesia epidural foi significativamente menor naquelas mulheres que haviam sido submetidas à prática de hipnose } \\
\text { ( } 28 \% \text { versus } 31 \% \text { de mulheres submetidas). }\end{array}$ \\
\hline $\begin{array}{c}\begin{array}{c}\text { Finlayson } \mathrm{K} \text {, et } \\
\text { al. }\end{array} \\
\text {. }\end{array}$ & 2015 & Caso-controle & $\begin{array}{l}\text { - A solicitação por anestesia epidural foi significativamente menor naquelas mulheres que haviam sido submetidas à prática de hipnose } \\
\text { ( } 72 \% \text { versus } 81 \% \text { de mulheres submetidas). }\end{array}$ \\
\hline Beevi Z, et al. & 2016 & Caso-controle & $\begin{array}{l}\text { - A solicitação por anestesia epidural foi signific ativamente menor naquelas mulheres que haviam sido submetidas à prática de hipnose } \\
\text { ( } 12,5 \% \text { versus } 30 \% \text { de mulheres submetidas). }\end{array}$ \\
\hline Steela A, et al. & 2016 & Coorte & $\begin{array}{l}\text { - Concluiu-se que mulheres adeptas à hipnose durante a gestação são mais propensas a utilizarem outras formas de medicina } \\
\text { complementar, como acupuntura e meditação. }\end{array}$ \\
\hline Waisblat $\mathrm{V}$, et al. & 2016 & Caso-controle & $\begin{array}{l}\text { - } \quad \text { Observou-se redução dos niveis de estresse ao longo da gestação. } \\
\text { - Foi observado maior controle emocional no caso de mudanças de plano no momento do parto. }\end{array}$ \\
\hline Beevi Z, et al. & 2017 & Caso-controle & $\begin{array}{l}\text { - Nenhuma alteração foi observada na duração das fases do trabalho de parto. } \\
\text { A solicitação por anestesia epidural foi significativamente menor naquelas mulheres que haviam sido submetidas à prática de hipnose } \\
\text { (4,3\% versus } 0 \% \text { de mulheres submetidas). }\end{array}$ \\
\hline Legrand $F$, et al. & 2017 & Caso-controle & balho de parto, não houve \\
\hline
\end{tabular}


QUADRO 1: RESULTADOS APRESENTADOS PELOS 10 ESTUDOS LEVANTADOS NA BUSCA DA LITERATURA.

\section{CONCLUSÃO:}

O estado mental natural "de consciência alterada" proporcionado pela hipnose tem inúmeras aplicações, inclusive no contexto da medicina. No contexto da obstetrícia, destaca-se o aumento da procura de alternativas para controle da dor no momento do parto e práticas que atuem positivamente no estresse e ansiedade tão comuns ao período gestacional.

Nesse âmbito, em relação ao período gestacional e ao momento do trabalho de parto, os estudos avaliados conseguiram demonstrar que a utilização de sessões de hipnose ao longo dos trimestres pode influenciar significativamente nos sintomas físicos, psicológicos e comportamentais vivenciados pelas mulheres. No momento do parto, a hipnose demonstrou impacto significativo no alívio da dor e controle de estresse. Nesse âmbito, esse tipo de intervenção evidencia-se com uma excelente complementação a medicina convencional, auxiliando essas mulheres ao longo do período gestacional.

Diante desse cenário, esse tipo de intervenção evidencia-se com uma excelente complementação a medicina convencional, auxiliando essas mulheres ao longo do período gestacional. E, por isso, reforça-se a necessidade de cada vez mais os serviços de saúde e profissionais das áreas tomarem conhecimento e oferecerem essa alternativa às mulheres.

\section{REFERÊNCIAS:}

1. MARTíN, MaríaÁngelesLudeña. Hipnose: Surpresa e Hipnoidal como Fatores da Mudança Terapêutica nos Distúrbios Emocionais. 2013. 251 f. Tese (Doutorado) Curso de Psicologia, Universidade de Coimbra Faculdade de Psicologia e de Ciências da Educação, Coimbra, 2013.

2. FINLAYSON, Kenneth et al. Unexpected consequences: women's experiences of a self-hypnosis intervention to help with pain relief during labour. Bmc Pregnancy And Childbirth, [s.l.], v. 15, n. 1, p.1-9, 25 set. 2015. Springer Nature. 
3. DOWNE, $S$ et al. Self-hypnosis for intrapartum pain management in pregnant nulliparous women: a randomised controlled trial of clinical effectiveness. Bjog: An International Journal of Obstetrics \&Gynaecology, [s.I.], v. 122, n. 9, p.1226-1234, 11 maio 2015.

4. BEEVI, Zuhrah; LOW, Wah Yun; HASSAN, Jamiyah. The Effectiveness of Hypnosis Intervention for Labor: An Experimental Study. American Journal Of Clinical Hypnosis, [s.l.], v. 60, n. 2, p.172-191, 11 set. 2017. Informa UK Limited.

5. WAISBLAT, V., Langholz, B., Bernard, F., Arnould, M., Benassi, A., Ginsbourger, F., Guillou, N., Hamelin, K., Houssel, P., Hugot, P., Martel-Jacob, S., Moufouki, M., Musellec, H., Nid Mansour, S., Ogagna, D., Paqueron, X., Zerguine, S., Cavagna, P., Bloc, S., Jensen, M. and Dhonneur, G. (2016). Impact of a Hypnotically-Based Intervention on Pain and Fear in Women Undergoing Labor. International Journal of Clinical and Experimental Hypnosis, 65(1), pp.64-85.

6. BEEVI, Zuhrah; LOW, Wah Yun; HASSAN, Jamiyah. Impact of Hypnosis Intervention in Alleviating Psychological and Physical Symptoms During Pregnancy. American Journal Of Clinical Hypnosis, [s.I.], v. 58, n. 4, p.368-382, 22 mar. 2016. Informa UK Limited.

7. STREIBERT, LA et al. Clinical Study: Change in Outlook Towards Birth After a Midwife Led Antenatal Education Programme Versus Hypnoreflexogenous SelfHypnosis Training for Childbirth GeburtshFrauenheilk 2015; 75: 1161-1166

8. WERNER, A; et al. Antenatal Hypnosis Training and Childbirth Experience: A Randomized Controlled Trial. BIRTH 40:4 December 2013 272-280

9. CYNA, et al. Hypnosis Antenatal Training for Childbirth: a randomised controlled trial. Bjog: An International Journal of Obstetrics \&Gynaecology, [s.I.], v. 120, n. 10, p.1248-1259, 3 jul. 2013. Wiley A. Steel et al. The characteristics of women who use hypnotherapy for intrapartum pain management: Preliminary insights from a nationallyrepresentative sample of Australian women Complementary Therapies in Medicine 25 (2016) 67-70

10. LEGRAND, F., Grévin-Laroche, C., Josse, E., Polidori, G., Quinart, H. and Taïar, R. (2017). Effects of hypnosis during pregnancy: A psychophysiological study on maternal stress. Medical Hypotheses, 102, pp.123-127. 
11. STEEL, A., Frawley, J., Sibbritt, D., Broom, A. and Adams, J. (2016). The characteristics of women who use hypnotherapy for intrapartum pain management: Preliminary insights from a nationally-representative sample of Australian women. ComplementaryTherapiesin Medicine, 25, pp.67-70. 